\title{
Rated Life Calculation Potential Of Gearbox Model Based Force Estimates
}

\author{
Jan Helsen ${ }^{1}$, Daniele Brandolisio ${ }^{1}$, Bart Peeters ${ }^{2}$, Frederik Vanhollebeke ${ }^{3}$,Joris Peeters ${ }^{3}$, David Moens ${ }^{4}$, \\ Wim Desmet ${ }^{1}$ \\ 1. Mechanical Engineering Department \\ KULeuven, \\ Celestijnenlaan 300 b bus 2420, Leuven, Belgium \\ 2. LMS International \\ Interleuvenlaan 68, Leuven, Belgium \\ 3. ZF Wind Power NV \\ Ottergemsesteenweg 439, Gent, Belgium \\ 4. Lessius Mechelen - campus De Nayer, Department of Applied Engineering, \\ Jan De Nayerlaan 5, B-2860, Sint-Katelijne-Waver, Belgium
}

\begin{abstract}
:
One main contributor for gearbox rated lifetime estimation is the assessment of bearing loading during predicted operating conditions. This paper investigates an approach to determine input bearing loading by means of a TPA (Transfer Path Analysis) approach. TPA is suggested to retrieve internal bearing forces from acceleration measurements acquired at the outside of the gearbox housing. However, classical TPA methods would require the gearbox to be dismantled during the transfer path determination process. This poses significant practical challenges. To overcome this issue, this paper investigates the possibility of using a flexible multibody simulation model to calculate the different frequency response functions between bearings forces and acceleration sensors. All simulations use a flexible multibody modeling approach, which has been extensively validated. Main results of this validation process have been published by the authors in the past. The paper discusses the results of such analysis on a multi-megawatt wind turbine gearbox. Here, simulated acceleration measurements on the gearbox housing are processed into bearing forces. The feasibility of using these forces for a rating life calculation is investigated.
\end{abstract}

Keywords: gearbox dynamics, multibody, transfer path analysis, model inversion, L-curve

\section{Introduction:}

High reliability and optimized noise and vibration behavior are important aspects of a good wind turbine design [1]. Bearing loading during operating conditions plays an important role in both fields [2,3]. Therefore, it is essential to accurately determine these loads. However, direct bearing load measurements pose significant challenges both from a measurement technology point of view as in the practical realization of the instrumentation inside the gearbox. The possible use of sensors on the outside of the gearbox housing such as accelerometers has several advantages: amongst others their easy installation, robustness to external influences and low price in comparison to internal instrumentation. Nonetheless, the challenges are shifted to the determination of the relationships between the different internal quantities and the signals measured on the outside of the system. This paper uses an approach based on transfer path analysis (TPA). However, classical TPA methods would require the gearbox to be dismantled during the transfer path determination process. This poses significant practical challenges. To overcome this issue, this paper investigates the possibility of using a flexible multibody simulation model to generate the necessary input information for the transfer path calculations.

Different drivers govern wind turbine gearbox behavior in the different frequency ranges of interest [4]. In this work three ranges of interest are defined: the quasi-static range, gearbox rigid dynamic range and gearbox flexible range. The gearbox behavior in the first frequency range is completely defined by the quasi-static response of the drivetrain to quasi-static external loading originating from the rotor and the generator. In the second frequency range the rigid body motions of the gearbox and other wind turbine drivetrain components are the main drivers of the drivetrain dynamics. The third frequency range shows highly complex modal behavior. Local gearbox flexibilities and local modal behavior are significant drivers in this frequency range. Since TPA uses the local gearbox modal behavior to link different quantities within a system this technique is advisable for the third frequency range. Therefore, the approach discussed in this paper will focus on bearing 
force estimation in the third frequency range. The paper ends with the study of the applicability of the identified loading histories for bearing rated life estimation.

\section{Methodology:}

The radial and axial loads acting on the internal bearings are identified during operating conditions by means of a system inversion method: a set of measured external operational response signals are combined with an inverted system model for the calculation of specific bearing forces. The inverted model can be frequency or time domain based. The pure gearbox housing model is used, without the presence of internal components to avoid terms that could result in coupling effects.

\subsection{Frequency domain technique}

To estimate the bearing forces a transfer path based approach (TPA) is used to retrieve the internal quantities. The model matrix consists of transfer functions between input bearing forces and output accelerations. Matrix inversion is applied to retrieve the input forces. To overcome the disassembling issue, a flexible multibody simulation model is used to calculate the different frequency response functions between bearings forces and acceleration sensors. The virtual models used in this investigation are based on previously validated models $[5,6,7]$.

The proposed method consists of the following steps:

1. Select the $\mathrm{n}$ bearing force degrees of freedom (DOFs) to investigate

2. Determine $2 * \mathrm{n}$ optimal acceleration positions on the gearbox housing. $2 * \mathrm{n}$ is suggested based to achieve a better pseudo inverse matrix.

3. Determine FRF matrix $\mathrm{H}$ between the bearing forces and chosen acceleration locations of the pure gearbox housing.

4. Calculate the pseudo inverse of the FRF matrix for each frequency of interest.

5. Measure acceleration signals at the selected response locations during operating conditions.

6. Multiply the inverted FRF matrix with the measured accelerations to calculate the bearing forces.

\subsection{Time domain technique}

In addition to the analysis in the frequency domain it is possible to perform a time domain based approach. The assumption made in this method is that the full dynamic behavior of the system is linear and fully known since a model is used to describe the system. This implies that a discrete-time state-space model of the system can be defined:

$$
\begin{gathered}
\dot{x}=\mathrm{Ax}+\mathrm{Bu} \\
\mathrm{y}=\mathrm{C} \mathrm{x}+\mathrm{Du}
\end{gathered}
$$

with $\mathrm{x}_{\mathrm{k}}$ the vector containing the states of the system, $\mathrm{u}_{\mathrm{k}}$ the vector of the externally applied forces and $\mathrm{y}_{\mathrm{k}}$ the vector with the resulting system responses. In the gearbox case the external forces applied to the forward model are all bearing and gear forces introduced in the gearbox housing.

Since the system matrices (ABCD) are fully known in the simulation model it is possible to analytically invert the system to obtain the inverse model. Writing this model again in state space notation as a function of a new $\mathrm{x}_{\mathrm{inv}}$ - vector containing the inverse states and the $\mathrm{u}_{\mathrm{inv}}$ - vector containing the accelerations on the gearbox housing results in:

$$
\begin{gathered}
x_{i n v}^{\cdot}=\left(\mathrm{A}-\mathrm{B} \cdot D^{-1} \cdot \mathrm{C}\right) x_{\mathrm{inv}}+\left(\mathrm{B} \cdot D^{-1}\right) u_{\mathrm{inv}} \\
y_{i n v}=\mathrm{D}^{-1} \cdot \mathrm{C} \cdot \mathrm{x}_{i n v}+\mathrm{D}^{-1} \cdot \mathrm{u}_{i n v}
\end{gathered}
$$




$$
\begin{gathered}
x_{i n v}^{\cdot}=\mathrm{A}_{i n v} x_{\mathrm{inv}}+\mathrm{B}_{i n v} u_{\mathrm{inv}} \\
y_{i n v}=C_{i n v} x_{\mathrm{inv}}+D_{i n v} u_{\mathrm{inv}}
\end{gathered}
$$

Time simulation using acceleration signals as input vector $\mathrm{u}_{\mathrm{k}}$ allows the calculating the force response vector at the bearing and gear force locations.

\section{Accuracy considerations}

Three main drivers determine the accuracy of both the frequency and the time domain approach: the system model accuracy, the model inversion error and the system observability corresponding to the used sensor set-up. The ability of the reduced model, in this case the gearbox housing, to describe the system dynamics determines the model accuracy. If experimental FRFs are used the main error sources are measurement errors. In the case of a model-based approach the quality of the experimental model update is the defining factor. Since the forward model linking accelerations to input forces is used, errors will be introduced by matrix inversion of the FRF matrix in case of the frequency based approach and D-matrix inversion for the time based approach. The third source of error is due to measuring only a reduced set of response points, which could result in bad observability of certain dynamic content of the system. The following paragraphs define an optimization approach for improving the quality of the matrix inversion and discuss the observability challenges.

\subsection{Observability and optimal sensor locations}

The dynamic response of the system is measured by means of acceleration sensors placed on the gearbox housing. If one is considering the state-space equations the state-space vector contains the number of independent variables needed to fully describe the state of the system. In practice however it is not possible to monitor all degrees of freedom. Therefore the vibration sensors should be placed on those locations that result in maximal observability. This paper suggests the use of the condition number of the system FRF matrix to choose the most optimal sensor locations. The condition number of a matrix is the ratio of the largest singular value to the smallest singular value of the matrix. It is a measure of the ill conditioning of the matrix. Reduction of condition numbers usually results in an improved force determination [8]. Literature review revealed that it is suggested to use at least twice the number of forces to estimate as number of response points [9]. This paper suggests a bootstrapping approach to choose the optimal locations. A net of virtual accelerometers is created on the surface of the gearbox housing. An initial accelerometer location needs to be selected from the virtual accelerometer net. Subsequently the algorithm adds additional accelerometers one by one until the total number of requested accelerometers is reached. For each time an accelerometer is added the influence of all virtual accelerometers on the total FRF matrix condition number is tested. All accelerometers of the virtual net are added one by one and the corresponding condition number of the FRF matrix recorded. The accelerometer corresponding to the minimum condition number is chosen as additional accelerometer and added to the set.

\subsection{Pseudo-inverse matrix calculation}

A critical step in both the frequency and time based approach is the inversion of the FRF matrix or system D matrix. Traditionally a pseudo inverse is calculated. The classical least-squares approach is used to calculate the pseudo inverse of the FRF matrix. Good conditioning of the FRF matrix is essential for high quality inversion. There are several influence factors for ill conditioning. Two or more excitations can be too close and are therefore difficult to separate due to too equivalent response. Another possibility is poor modal participation of the response particularly in low frequency or in the vicinity of lightly damped resonant frequencies of the structure. The number of modes significantly participating to the response has to be at least equal to the number of unknown forces. In the case of ill-conditioning some linear dependencies are introduced between columns of the transfer matrix. Several linear combinations exist, which could generate the measured deflection shapes with equivalent residual quantities. Therefore one of these solutions has to be chosen. The strict application of the classical least-squares approach would lead to the selection of the solution with the smallest residue, but the magnitude of this solution is often too large to be realistic $[8,10]$. Many solutions with significantly higher residue are far more realistic. Different methods exist. The most popular regularization methods are truncated singular value decomposition (TSVD) and Tikhonov [10]. These methods are based on the adjustment of a regularization parameter, that changes the importance given to the minimization of the residue norm on the one hand and the solution norm on the other hand. Many variants of this method are available. This document discusses an approach based on the L-curve principle [11]. The L-curve principle is combined with a TSVD regularization, based on the singular value decomposition of the transfer matrix: 


$$
[H]_{m n}=[U]_{m n}[S]_{n n}[V]_{n n}^{*}
$$

With:

- $\mathrm{H}$ : FRF Matrix

- $\mathrm{M}$ : number of outputs (response points)

- $\quad \mathrm{N}$ : number of inputs

- $\mathrm{S}$ : diagonal matrix of singular values sorted in descending order

- $\mathrm{V}$ : unitary matrix

- $\mathrm{U}$ : matrix such that $\mathrm{U}^{*} \cdot \mathrm{U}=\mathrm{I}$

The TSVD is based on an approach to regularize the matrix $[S]_{n n}^{-1}$ by zeroing the last elements of the diagonal matrix. A regularizing parameter s corresponding to the number of kept singular values is defined. (n-s) singular values are set to zero in this approach. Since the elements of S are arranged in descending order this corresponds to the (n-s) smallest singular values. The regularized pseudo-inverse of the transfer matrix based on the chosen s number can be written as follows:

$$
[H]_{m n}^{+}=[V]_{n n}\left[\begin{array}{cc}
{[S]_{s S}^{-1}} & 0 \\
0 & 0
\end{array}\right][U]_{m n}^{*}
$$

The parameter $\mathrm{s}$ is the artificial rank given to the transfer matrix. In case the system matrix is rank deficient the parameters $\mathrm{s}$ should be chosen such that the solution of the above equation is of minimal norm. The selection is done based on the Lcurve principle adapted for TSVD discussed in [10]. The selection is based on the parametric curve with the residue norm $(\rho)$ on the $x$-axis and the solution norm $(\eta)$ on the $y$-axis in function of the regularization parameter s. Figure 1 shows an example of such an L-curve.
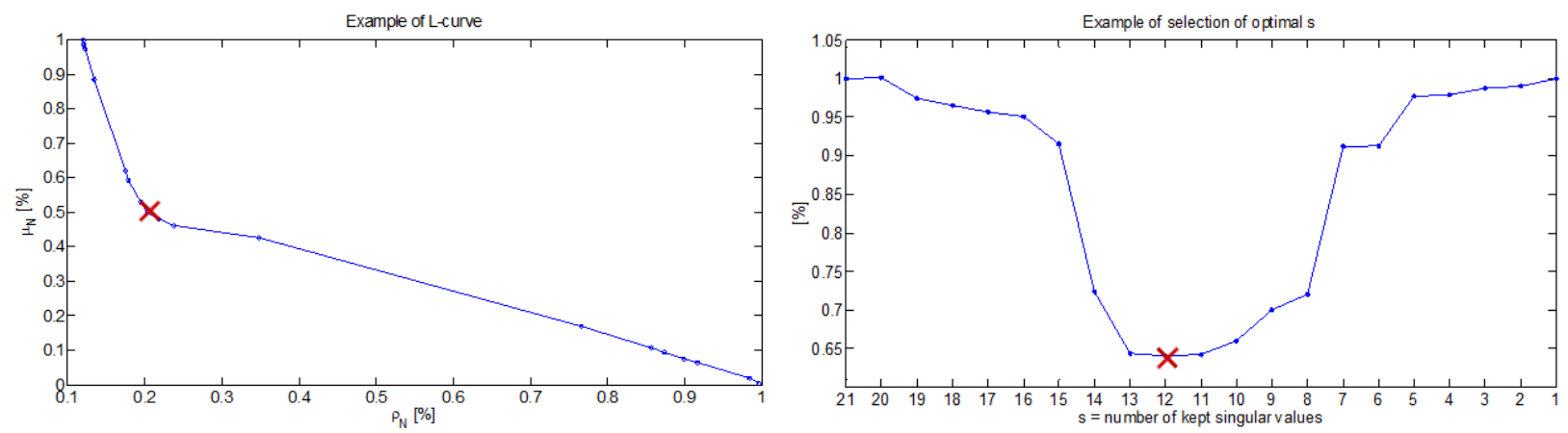

Fig. 1 Example of L-curve and corresponding parametric curve

The inputs for the L-curve are defined by:

$$
\begin{gathered}
\rho_{N}(s)=\frac{\|X-[H] F\|}{\|X\|} \\
\mu_{N}(s)=\frac{\mu(s)}{\mu(n)}
\end{gathered}
$$

$\rho_{N}(s)$ and $\mu_{N}(s)$ are the values of the solution norm and the normalized residue norm for $\mathrm{s}=\mathrm{n}$. This is the case of no regularization. The equation below uses these parameters to determine the optimal point on the L-curve[10]:

$$
\varepsilon(s)=\rho_{N}(s)+\mu_{N}(s)\left(1-\rho_{N}(n)\right)
$$


The optimal point of the L-curve is its angle for which the decrease of $s$ has caused an important diminution of the solution norm $\eta$ without a significant growth of the residue norm $\rho$. For the L-curve shown in the figure above the red cross marks the optimal point.

\section{Virtual models}

A representative multi-megawatt wind turbine gearbox is chosen for the feasibility study. Two models are used: a full gearbox flexible multibody model and a gearbox housing Craig Bampton Reduced finite element model. The former model is used to generate reference signals, whereas the latter model is used in the actual TPA calculations. Figure 2 shows the layout of the flexible multibody model of the entire wind turbine gearbox. The bearings under investigation are highlighted. The gearbox consists of three stages: a High Speed Stage containing the High Speed Shaft (HSS) with bearings HSS_B1 and HSS_B2, and the Low Speed Shaft (LSS) with bearings LSS_B1 and LSS_B2; A planetary Intermediate Speed Stage with one bearing PS2_B1; A planetary Low Speed Stage with bearings PS1_B1 and PS1_B2.

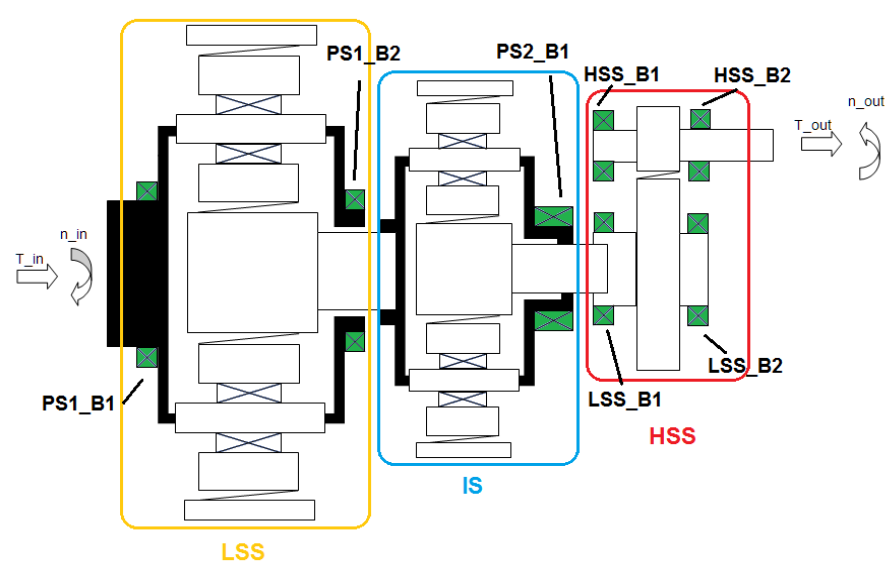

Fig. 2 Gearbox scheme and bearings locations (green)

On the outer surface of the gearbox model, 205 virtual tri-axial accelerometers are placed in a grid pattern, thus to cover the entire surface of the housing and capture the most information to allow for a future obeservability study.

\section{Virtual experiment}

To assess the potential of the time and frequency based TPA method a virtual experiment is conducted. For brevity reasons only the frequency based approach is discussed. The full gearbox model is used to generate time signals for all bearing forces of interest and accelerometers of the virtual accelerometer net. Nominal torque is applied to the planet carrier at the gearbox input. A rotational damper at the HSS output shaft creates steady state conditions after an initial run-up. Dynamic excitation is originating from the gear meshing at the different gear stages. Two seconds of signal length are cut from these steady state conditions and used as input for the TPA calculations. The input acceleration signals for the TPA are a subset of 63 acceleration signals. This number of sensor signals is equal to three times the number of bearing forces to estimate. Figure 3 shows an example bearing force frequency spectrum for TPA estimation and full gearbox model time simulation. Forces are compared at the different gear meshing frequencies, since excitation is necessary to result in a representative acceleration signal on the housing. In general it can be concluded that estimate quality changes over frequency. The errors can be due to observability and model inversion issues. Estimation tended to be worse at lower frequencies, which is in line with the higher condition numbers found in the low frequency range. Strain signals could be added to the measurement set to improve this low frequency behavior. Further investigations will focus on improving these condition numbers and trying to optimize observability. 


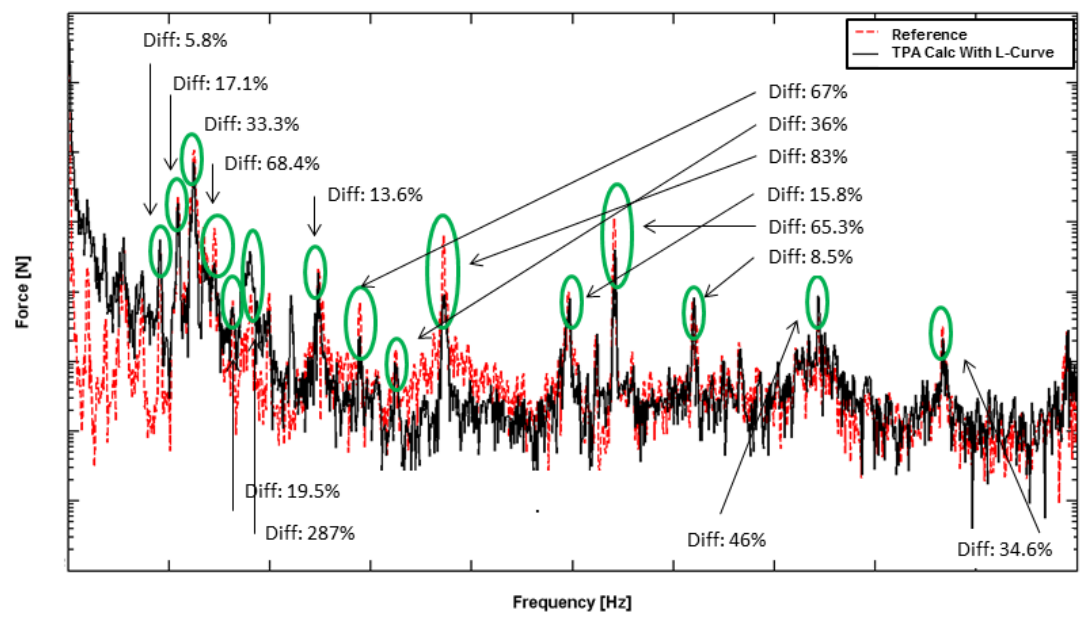

Fig. 3 Example of estimated bearing forces

\section{Potential for bearing rated life calculations}

A common calculation method for rated life of bearings is the $1_{10^{-}}$approach. Bearing life is expressed as the number of revolutions a bearing can achieve with $10 \%$ failure probability [12]. The input for these calculations is a bearing force time signal for the axial and two radial DOFs. In a first step these forces are classified in load bins depending on their value. These bin values for axial and radial DOFs are used as input to determine the total loads in the different DOFs and combine these loads into a total equivalent load:

with:

$$
P_{e q}=X F_{r}+Y F_{a}
$$

- $F_{r}$ : Radial force

- $\quad F_{a}$ : Axial force

- $X, Y$ : Specific bearing coefficients from catalogue

- $P_{e q}=$ equivalent dynamic bearing load

Including all equivalent bearing loads in the following formula results in an estimate of the rated life:

with:

$$
L_{10}=\left(\frac{C_{D}}{P_{e q}}\right)^{p}
$$

- $C_{D}=$ dynamic load rating (from catalogue)

- $\quad p=3$ for ball bearings, $10 / 3$ for roller bearings

There are several challenges with using the $\mathrm{L}_{10}$-approach in combination with the TPA approach. The first is the need for time-based signals. This challenge can be overcome by using the inverse FFT to transform the signals back to the timedomain. Since the bins-approach is used there is no need to save the exact moment in time that a peak occurred. The second main challenge is the quasi-static character of the $\mathrm{L}_{10}$-approach. The dynamic forces shown in the TPA spectra are superimposed on the quasi-static force fluctuations. Due to their low amplitude they are not visible in the force bins. Nonetheless there is a large number of cycles related to these loading conditions. To be able to objectively include their influence on the overall rated bearing life it would however be necessary to adjust the $\mathrm{L}_{10}$ approach. Since the TPA approach delivers the full frequency spectrum this extension is needed in order to fully use the potential of the TPA. 


\section{Conclusions}

This paper discussed a TPA based approach to determine bearing forces of a wind turbine gearbox by means of external acceleration measurements on the housing. The different challenges related to the estimation were suggested and a full method was described. The compatibility of this approach with classical $\mathrm{L}_{10}$ rated bearing life discussed and concluded that an extension of the classic formulae is needed if the non-quasi static forces identified by the TPA want to be included.

\section{Acknowledgements}

The IWT Flanders within the ALARM, Prognostics for Optimal Maintenance 2 and OptiWind projects. The IWT Flanders PROTEUS mandate of Jan Helsen.

\section{References}

[1] Aberrazzaq M.A., Hahn B., Analysis of the wind turbine standstill for a grid connected wind farm (case study), Renawable Energy, Vol. 33, pp. 89-104, 2006

[2] Errichello R., Muller J., Gearbox Reliability Collaborative Gearbox 1 Failure Analysis Report, NREL Report, NREL/SR-5000-53062, 2011

[3] Guo Y., Keller J. , LaCava W., Combined Effects Of Gravity, Bending Moment, Bearing Clearance and Input Torque On Wind Turbine Planetary Gear Load Sharing, AGMA Fall Technical Meeting, Dearborn, Michigan, 2012

[4] Helsen J., Marrant B., Vanhollebeke F., De Coninck F., Berckmans D., Vandepitte D., Desmet W., Assessment of excitation mechanisms and structural flexibility influence in excitation propagation in multi-megawatt wind turbine gearboxes: Experiments and flexible multibody model optimization, Mechanical Systems and Signal Processing, Vol. 40(1), pp.114-135, 2013

[5] Peeters J., Simulation of dynamic drive train loads in a wind turbine, PhD Dissertation, KULeuven, Belgium, https://repository.libis.kuleuven.be/dspace/handle/1979/344, 2006,

[6] Vanhollebeke F., Helsen J., Peeters J., Vandepitte D., Desmet W., Combining multibody and acoustic simulation models for wind turbine gearbox NVH optimisation, Proceedings of ISMA 2012, Leuven, 2012

[7] Helsen J., The Dynamics of High Power Density Gear Units with Focus on the Wind Turbine Application, PhD

Dissertation, KULeuven, https://lirias.kuleuven.be/handle/123456789/337483, Belgium, 2012

[8] A.N.Thite,D.J.Thompson: Selection of response measurement locations to improve inverse force determination, Applied Acoustics, Vol.67, p.797-818,2006

[9] Gajdatsy P.A., Advanced Transfer Path Analysis Methods, Dissertation, KULeuven, https://lirias.kuleuven.be/handle/123456789/289454, Belgium, 2011

[10] Leclère Q., Pezerat C., Laulagnet B., Polac L., Indirect measurement of main bearing loads in an operating diesel engine, Journal of Sound And Vibration, Vol. 286, pp. 341-361, 2005

[11] Hansen P.C., The 1-curve and its use in the numerical treatment of inverse problems, http://citeseer.nj.nec.com/196586.html

[12] ISO 281 Standard: Dynamic load ratings and rating life 